\title{
Research on E-commerce Credit Management System based on the Model of Multidimensional Credibility
}

\author{
Liu Bo
}

China West Normal University, Institute of foreign languages, Sichuan, Nanchong, China

Keywords: E-commerce; model of multidimensional credibility; credit scoring

\begin{abstract}
The traders in C2C e-commerce model are all the entities which are scattered and do not know each other, and there are more transaction security risks. If we want to reduce the risk of trading, we need to introduce the credit calculation in e-commerce. Although the current credit management system has more mature development in many e-commerce websites, aiming at the credibility of the users' preferences and credibility expressive methods it is not very perfect. Therefore, we construct the thought of multidimensional credibility management system and we provide the users with more flexible credibility degree for the corresponding potential partners which is calculated by aiming at their preferences in the paper, and at the same time we build the expressive methods of various credibility degrees so as to let the users be able to choose their favorite expressive ways according to their own usage habits, thus they can understand the credible degree of potential trading partners more clearly.
\end{abstract}

\section{Introduction}

Aiming at the existing problems and insufficiencies in the current credit management system we put forward the thought of the multidimensional (price, quality, service) credit management system in the paper. We can use the multidimensional credibility degree to replace the current single credit, and according to the users' preferences we design the weight of each dimension, and finally we form the credibility degree of transaction object which is dynamic and can reflect the users' preferences, thus it can provide a reference for the users' decisions in the network transaction. With the theory of cognitive psychology we explore the proper credibility dimension, and the size of the dimension is related to the efficiency of users' feedback scores. We apply the famous AHP method in the decision science to design and reflect the weight of credibility in each dimension about the users' preferences, thus we form the complete design of multidimensional credit scoring model. Then we use J2EE to develop the multidimensional credibility evaluation model, and do the actual analysis in the paper.

\section{Multidimensional Scoring Calculation}

Having an overall consideration of the current credit management system, the author analyzes that the model aggregated by the credibility can improve some problems in credit management system in the paper. In addition, the problems which are improved by it are mainly the multidimensional rating problems of credibility. And the dimensions of credibility mainly depend on the websites like e-commerce or the users' requirements.

1. Scores of Aggregating Each Credibility Dimension. The formula which is aggregated by each credibility dimension. 


$$
R_{m}=\frac{\sum_{g=1}^{m} r_{m} \times W\left(R_{i}^{\text {other }}\right)+S}{n} n=1,2, \ldots \ldots I=1,2, \ldots \ldots m
$$

In the entity after we obtain the nth evaluation we obtain the cumulative score of $i$ this dimension, $R_{m}$. In the kth transaction activity we obtain the credibility evaluation of $\mathrm{i}$ this dimension, $R_{k}$. In the transaction, the credit score combined by the party who participates in the evaluation is $R_{k}{ }^{\text {other }}$, and the corresponding weight is S. If the value of $R_{k}{ }^{\text {other }}$ is higher, then the degree of belief made by the people who participate in the evaluation is higher, so the weight which it evaluates will be bigger. If we need to determine the weight, we first should set $R_{k}$ as the credit score with combined evaluation in the kth transaction when the evaluation party releases the credit evaluation. The upper limit of this value is $\mathrm{D}$. After we carry out the standardized treatment on it, we can obtain $R_{k} / \mathrm{D}$ in $(0,1)$. We make $E_{k}=R_{k} / D$ as the weight which can reflect the size of the combined credit scores. We can make the formula(8)be simplified as

$$
R_{m}=\frac{\sum_{k=1}^{m} R_{k} \times E_{k}+S}{n} n=1,2, \ldots \ldots i=1,2, \ldots \ldots m
$$

In the formula mentioned above, the credit score of entity in the beginning is s. Generally it is 0 . After we complete the above analysis, we can know that if the credit score in the beginning is too low, it will influence the new website users to join in. Therefore, when we design it, we need to find the best initial value of credibility.

2. Scores of Aggregating Combined Multidimensional Credibility. Standing at in the importance degree perspective, the users make a comparison of the transaction objects selected by themselves. This is the thought of AHP. In this thought, $a_{y}$ is the importance of the ith dimension and the jth dimension, and it is also considered as the ratio of the credibility weight $w_{i}$ of the ith dimension and the credibility weight $w_{j}$ of the jth dimension, that is to say, $w_{i} / w_{j}$ is the ratio of the credibility weight of it and the jth dimension. When the credibility of each dimension is compared to each other we can obtain the following result.

$$
A_{m}\left[\begin{array}{cccc}
a 11 & a 12 & \ldots \ldots & a_{1 m} \\
a 21 & a 22 & \ldots . . & a_{2 m} \\
\cdot & \cdot & \cdot & \cdot \\
\cdot & \cdot & \cdot & \cdot \\
\cdot & \cdot & \cdot & \cdot \\
a_{m 1} & a_{m 2} & \ldots . . & a_{m m}
\end{array}\right] \approx\left[\begin{array}{cccc}
w_{1} / w_{1} & w_{1} / w_{2} & \ldots \ldots & w_{1} / w_{m} \\
w_{2} / w_{1} & w_{2} / w_{1} & \ldots . . & w_{2} / w_{m} \\
\cdot & \cdot & \cdot & \cdot \\
\cdot & \cdot & \cdot & \cdot \\
\cdot & \cdot & \cdot & \cdot \\
w_{m} / w_{1} & w_{m} / w_{2} & \ldots . . & w_{m} / w_{m}
\end{array}\right]
$$

We set all-vector $\mathrm{W}=\left(w_{1}, w_{2} \ldots \ldots w_{m}\right)^{r}$, to meet the conditions $\sum_{i=1}^{m} w_{1}=1$ 且 $w_{i} \succ 0, m=1,2 \ldots \ldots$, then we have

$$
A W=\left[\begin{array}{cccc}
w_{1} / w_{1} & w_{1} / w_{2} & \ldots \ldots & w_{1} / w_{m} \\
w_{2} / w_{1} & w_{2} / w_{1} & \ldots . . & w_{2} / w_{m} \\
\cdot & \cdot & \cdot & \cdot \\
\cdot & \cdot & \cdot & \cdot \\
\cdot & \cdot & \cdot & \cdot \\
w_{m} / w_{1} & w_{m} / w_{2} & \ldots . . & w_{m} / w_{m}
\end{array}\right]\left[\begin{array}{l}
w_{1} \\
w_{2} \\
\cdot \\
\cdot \\
\cdot \\
w_{m}
\end{array}\right]=m\left[\begin{array}{l}
w_{1} \\
w_{2} \\
\cdot \\
\cdot \\
\cdot \\
w_{m}
\end{array}\right]
$$


Therefore we apply the method of eigenvector,

$(A-n I) \quad W \approx 0$

(3).

In the formula above, I is the identity matrix. If we have a correct estimation on $\mathrm{A}$, then we have the above formula(3) $=0$. In the homogeneous linear equations, $\mathrm{W}$ only has the trivial solution. If $\mathrm{A}$ can't make the below formula(4) =0, then the small perturbation elements in A will appear, and it also represents that the eigenvalue will have small perturbation. Therefore, we obtain the following formula.

$$
A W=\lambda_{\max } W
$$

Among them, the largest eigenvalue in $\mathrm{A}$ is in $\mathrm{A} \lambda_{\max }$, and in formula(4)we can obtain $\mathrm{W}$. That is to say, in formula(4)we can calculate the weight of credibility in each dimension, so the obtained weight of credibility in each dimension can also obtain the users' multidimensional combined credit score.

The expression form of aggregating the combined credit is shown as follows.

$$
R_{n}=\left[\begin{array}{cc}
\sum_{i=1}^{m} R_{1 n \times w 1} & n \leq M \\
\sum_{i=1}^{m} R_{i(n-M+1)} \times W_{1} & n \succ M
\end{array}\right]
$$

In the above formula, the combined credit score obtained from the initial evaluation to the nth evaluation is $R_{n}$. In the combined credibility, the weight which is opposed by i-dimensional credibility is $W_{i}$. The weights of credit scores with various dimensions represent each user's different preferences.

Some entities with worse or better behaviors use all the informations and make them feedback. The differences between the combined credit scores about the feedback of informations which have been obtained recently are not so big. However, when we calculate the combined credibility, we should pay attention to the feedback informations which have been obtained recently, and the behavior is more reasonable. In Bizltate. Com, when its system of credit management calculates the combined credibility, it only calculates the information within 90 days in recent days. In formula(5), $\mathrm{M}$ is the threshold. The value is determined by the features of websites like the e-commerce, and the threshold shall prevail. So in this way we can calculate the score of combined credibility.

3. The Weight of Confirming Each Dimension. We give the scores of the feedback value in detail from the three dimensions (price, quality, service). When an user choose the transaction partner, we can see the transaction partner's credit representing graph of each dimension. At the same time, we can also endow the different weights to different dimensions according to our own preferences so as to form the combined credibility which can reflect the user's preferences.

Standing at in the importance degree perspective, the users make a comparison of the transaction objects selected by themselves. This is the thought of AHP. In this thought, $a_{i j}$ is the more important expected value of the ith credibility in the 1st credibility, and it is considered as the credibility weight $w_{i}$ of the 1 st dimension. $w_{i} / w_{j}$ is the ratio of it and the credibility weight $w_{j}$ in the jth dimension. At present the individual who wants to conduct a transaction with the user is $\mathrm{L}$. We use the matrix A to represent the results with the pairwise comparison of 3-dimensional credit (in order they are price, quality and service) preferences.

$$
A=\left(\begin{array}{lll}
a_{11} & a_{12} & a_{13} \\
a_{21} & a_{22} & a_{23} \\
a_{31} & a_{32} & a_{33}
\end{array}\right) \approx\left(\begin{array}{lll}
w_{1} / w_{1} & w_{1} / w_{2} & w_{1} / w_{3} \\
w_{2} / w_{1} & w_{2} / w_{2} & w_{2} / w_{3} \\
w_{3} / w_{1} & w_{3} / w_{2} & w_{3} / w_{3}
\end{array}\right)=\left(\begin{array}{ccc}
1 & 1 / 3 & 1 / 2 \\
3 & 1 & 3 \\
2 & 1 / 3 & 1
\end{array}\right)
$$

In other words, $L$ thinks that the quality is three times more important than the price and service, and the importance of service is two times higher than the price. From this we know that this individual's degree of placing the importance on the service, quality and price of the products from 
high to low, in order, should be quality, service and price.

We set the vector $W=\left(w_{1}, w_{2}, w_{3}\right)^{T}$, and we make it meet the conditions $\sum_{i=1}^{3} w_{i}=1$ and $\mathrm{w}_{\mathrm{i}}>$ $0, \mathrm{i}=1,2,3$, then we have

$$
A W=\left(\begin{array}{lll}
w_{1} / w_{1} & w_{1} / w_{2} & w_{1} / w_{3} \\
w_{2} / w_{1} & w_{2} / w_{2} & w_{2} / w_{3} \\
w_{3} / w_{1} & w_{3} / w_{2} & w_{3} / w_{3}
\end{array}\right)\left(\begin{array}{l}
w_{1} \\
w_{2} \\
w_{3}
\end{array}\right)=3\left(\begin{array}{l}
w_{1} \\
w_{2} \\
w_{3}
\end{array}\right)
$$

Therefore we have

$$
(A-3 I) W \approx 0(6)
$$

We apply the method of eigenvector. In the formula(6), I is the identity matrix. If we have a correct estimation on $\mathrm{A}$, then we have the above formula(6) $=0$. In the homogeneous linear equation, $\mathrm{W}$ only has the trivial solution. If A can't make the below formula(7) $=0$, then the small perturbation elements in A will appear, and it also represents that the eigenvalue will have small perturbation. Therefore, we obtain the following formula.

$$
A W=\lambda_{\max } W
$$

Among them, the largest eigenvalue in $\mathrm{A}$ is in $\mathrm{A} \lambda_{\max }$, and in above formula we can obtain $\mathrm{W}$ ( the weight of credibility in each dimension). Finally we can obtain the the score of aggregating the multidimensional combined credibility. We calculate the weight of each dimension, and we have known the given matrix A. According to the formula(5),we can obtain the determinant.

$$
\left|\begin{array}{ccc}
1-\lambda & 1 / 3 & 1 / 2 \\
3 & 1-\lambda & 3 \\
2 & 1 / 3 & 1-\lambda
\end{array}\right|=0
$$

We calculate the value of $\lambda_{\max }$, namely3.0536,therefore

$$
\left(\begin{array}{ccc}
-2.0536 & 1 / 3 & 1 / 2 \\
3 & -2.0536 & 3 \\
2 & 1 / 2 & -2.0536
\end{array}\right)\left(\begin{array}{c}
w_{1} \\
w_{2} \\
w_{3}
\end{array}\right)=0
$$

By calculating the homogeneous linear equations, we calculate the weight vector. And it is $W^{T}=(0.1571,0.5936,0.2493)$

In other words, A thinks that the corresponding weights of credibility in each dimension about the price, quality and service should be $0.1571,0.5939$ and 0.2493 respectively.

4. Combined Result of Aggregating Multidimensional Credibility. Based on the given credit value in each dimension mentioned above, we can calculate the users' combined multidimensional credibility which can reflect the users' preferences next so as to provide a more scientific and reasonable reference for the users when the users choose the transaction objects.

According to the combined credibility, the unified expression is aggregated.

$$
R_{25}=\sum_{i=1}^{3} R_{i, 25} \times w_{1}
$$

We calculate the seller's combined credibility in the K store which can reflect L user's preference. And the results are shown in Table 1. 
Table 1 Combined Multidimensional Credibility Degree

\begin{tabular}{|c|c|c|}
\hline $\begin{array}{c}\text { Sequence } \\
\text { Number }\end{array}$ & feedback time & Combined Credibility \\
\hline 1 & 2008.12.01 & 0.055 \\
\hline 2 & 2008.12 .14 & 0.2055 \\
\hline 3 & 2009.01.10 & 0.2228 \\
\hline 4 & 2009.02.27 & 2.2812 \\
\hline 5 & 2009.04 .18 & 0.3917 \\
\hline 6 & 2009.06 .01 & 0.6166 \\
\hline 7 & 2009.06 .25 & 0.7309 \\
\hline 8 & 2009.07 .18 & 0.7435 \\
\hline 9 & 2009.10 .01 & 0.8683 \\
\hline 10 & 2009.10 .15 & 0.9023 \\
\hline 11 & 2009.10.26 & 0.913 \\
\hline 12 & 2009.11 .15 & 1.3981 \\
\hline 13 & 2009.11 .18 & 1.4073 \\
\hline 14 & 2009.11 .21 & 1.9311 \\
\hline 15 & 2009.11 .29 & 2.1829 \\
\hline 16 & 2009.11 .29 & 2.6417 \\
\hline 17 & 2009.12 .30 & 2.6656 \\
\hline 18 & 2010.02 .27 & 2.8462 \\
\hline 19 & 2010.02 .27 & 3.0252 \\
\hline 20 & 2010.03 .01 & 3.2358 \\
\hline 21 & 2010.03 .22 & 3.3904 \\
\hline 22 & 2010.01 .30 & 3.9323 \\
\hline 23 & 2010.03 .06 & 3.9349 \\
\hline 24 & 2010.03.11 & 4.1325 \\
\hline 25 & 2010.04 .20 & 4.3097 \\
\hline
\end{tabular}

\section{Conclusion}

Aiming at the existing problems and insufficiencies in the current credit management system we put forward the thought of multidimensional credit management system in the paper. We can use the multidimensional credibility degree to replace the current single credit, and according to the users' preferences we design the weight of each dimension, and finally we form the credibility degree of transaction object which is dynamic and can reflect the users' preferences, thus it can provide a reference for the users' decisions in the network transaction. With the theory of cognitive psychology we explore the proper credibility dimension, and the size of the dimension is related to the efficiency of users' feedback scores. We apply the famous AHP method in the decision science to design and reflect the weight of credibility in each dimension about the users' preferences, thus we form the complete design of multidimensional credit scoring model. We improve the commonly used credibility numerical expressive method, and design the evolutive graphical representation of multidimensional credibility out. So it can clearly reflect the corresponding changes situation of time and credibility. Thus, it can let the users be able to know the transaction partners' credibility in each stage with more detailed attitudes.

\section{References}

[1]Zhang Jingan, Liu Jun. Research on the Evaluation Model of Customer's Trust Degree in E-commerce Market_—Based on the Consumer-to-consumer E-commerce[J]. China Circulation 
Economics, 2009, 02:35-38.

[2]Shi Yanxia, Ni Ling, Guan Guangyang. Research on the Competitiveness Evaluation Index System of E-commerce Websites_—Based on the Perspective of Resources Field Force[J]. Books and Information Work, 2009, 02:129-133.

[3]Su Fangli. Research on E-commerce Lemon Problem based on Dynamic Game Model [J]. Books and Information Work, 2009, 10:140-144.

[4]Song Guangxing, Cao Chunfang. Research on Online Reputation Score aggregating approach in E-commerce and Personalized Recommendation[J]. Forecast, 2009, 05:55-58+64.

[5]Shao Jiabing, Luo Xuan, and Yang Haifeng. The Influence of C2C E-commerce Disputes on the Transaction Evaluation Results[J]. Statistics and Decision, 2009, 19:101-103.

[6]Wang Yangyang. Exploration of C2C Credit Evaluation Model [J]. Journal of Shanxi University of Finance and Economics, 2010, S1:70+95.

[7]Li Shengqi, Chen Kui. Research on Consumer-to-consumer E-commerce Websites Credit Evaluation Model[J]. China Business and Market, 2010, 06:61-64.

[8]Wu Kewen, Zhao Yuxiang and Zhu Qinghua. Research Review on the Seller's Credit Evaluation in the C2C E-commerce[J]. Books and Information Work, 2010, 14:136-139+101.

[9]Wang Xuan, Guo Weichen. Analysis and Research on the Third Party Logistics Enterprises' Credit Evaluation[J]. Development and Research, 2011, 04:129-133. 